\title{
Positioning Reference Signal-Orientated Moving Train Localization in Ricean Channel
}

\author{
Cui-Ran Li and Jian-Li Xie \\ School of Electronics and Information Engineering, Lanzhou Jiaotong University \\ jianli.xie@yahoo.com
}

\begin{abstract}
In this paper, the challenging problem of train localization is investigated. Precise localization of rail vehicles is a key element for development of more efficient and safe operation of high-speed railway. The localization task is formulated in a 3GPP LTEbased framework that enables us to derive the Doppler frequency offset of moving train, by exploitation of positioning reference signal (PRS) placed in the subframe, in Ricean channel. Then, a PRS-oriented train position approach is proposed. The PRS time difference of arrival from positioning references eNB1 and eNB2, is calculated by sequences cross correlation, and the train running curve is determined by inquiring about the line database of track section. Finally, the train position is evaluated by combining the information of PRS time difference of arrival and the train's moving trajectory. In the simulation, the localization performance is well demonstrated, when two types of railway alignments, i.e., straight line and circle curve, are considered.
\end{abstract}

Keywords: Positioning reference signal, train localization, LTE, railway alignment, positioning accuracy.

\section{Introduction}

The world's railway infrastructure has been experiencing substantial growth in the last two decades. This growth imposes great pressure on rail operators due to the need to ensure the safety of rail transportation. The traditional wired train localization systems [1] are based mainly on trackside infrastructure elements like interlockings, signals, axle counters, and balises to ensure the safe operation of railways. This requires large investments. However, many railway lines suffer from low profitability, which makes it difficult to justify high investments into the infrastructure. Even though Global Positioning System (GPS) can achieve high localization accuracy and has been widely used in vehicular systems including railways but are subject to multiple limitations. For example, trains in subway systems primarily operate underground. Even in the aboveground railways, trains may frequently pass through GPS-dark territories such as tunnels, hilly regions, or urban canyons [2].

One way to solve about problem is to develop new train protection systems that require less trackside infrastructure. The development of railway wireless communication system allows replacing balise-based communication between the train and the inter-locking by cheaper wireless techniques. With the fast developing of High-Speed Railway (HSR) in China, current systems, such as GSM-R which only supports a maximum data rate of 200kbps [3], cannot meet the exploding demands for multi-media access while traveling. Much effort has been put on designing an advanced communication system in such extreme environment, and developing the overall working capabilities of newly proposed standards like LTE-R [4].

In this paper, a novel positioning reference signal (PRS)-orientated moving train localization method in Ricean channel has been proposed to promote the positioning precise in high-speed railway system. Differing from the traditional positioning schemes, 
the proposed method makes use of the LTE-R wireless communication network to fulfill the train position evaluation.

The rest of the paper is organized as follows. Section 2 is dedicated to an overview of work related to PRS-orientated localization algorithm. Section 3 presents the system model of our PRS-oriented train position approach. Experimental results and discussion are provided in Section 4. Finally, conclusions are summarized in Section 5.

\section{Related Work}

LTE adopted orthogonal frequency division multiple access (OFDMA) technique for the downlink (DL) transmission. In LTE Release 9 [5], the positioning reference signal (PRS) is introduced, which enables the user equipment (UE) to measure the reference signal time difference (RSTD) between different cells. The UE observes the PRS from different source transmitters (i.e. cellular base stations) in the neighborhood and makes certain measurements. Examples of such measurements include observed time difference of arrival (OTDOA) [6], which is the relative timing difference between a neighbor cell and the reference cell.

In the last few years, many efforts have been performed on positioning technology in LTE systems, and the current research concerning OTDOA mainly focused on commercial application. In [7], a research based on real measurements of OTDOA in the LTE $20 \mathrm{MHz}$ bandwidth using three separate base station sites in a realistic deployment is carried out, the impact of real measured propagation channels on OTDOA positioning is investigated. In 222.pdf, a novel OTDOA positioning scheme in heterogeneous LTEAdvanced systems is proposed, which can avoid the interference in heterogeneous LTEAdvanced systems and greatly improve the positioning accuracy. In [9], cooperative positioning in LTE systems was studied. In the proposed technique, OTDOA measurement method is used for UE-eNB connections, while RTT measurement method is used for UE-UE connections.

Moreover, [10] analyze and evaluate the location accuracy by using OTDOA techniques with three different path loss models such as fixing Algorithm, COST231Okumura-Hata and COST231-Walfisch-Ikegami. In the railway environment, one of the most challenging problems of train localization is the severe Doppler effects caused by a tremendous speed of up to $500 \mathrm{~km} / \mathrm{h}$ or so. Therefore, there is a strong requirement to evaluate the Doppler effects. Current work concerning Doppler estimation pays more attention to the direct DFS estimate in OFDM systems [11, 12]. It has been pointed out that multipath effects in high-speed railway channels are not so serious, due to the dominant existence of line-of-sight (LOS) components [12].

For our work, we address the problem of high-speed train localization in Ricean channel which highlights the effect of LOS component. The Doppler frequency offset of moving train is derived by exploitation of the PRS placed in subframes. Then, a PRSoriented train position approach is proposed. Finally, the train position is evaluated by combining the information of PRS time difference of arrival and the train's moving trajectory.

\section{System Model}

\subsection{Moving Train and Frame Configuration}

Consider a fast moving train with one radio transceiver (antenna) on each carriage, which will receive the downlink signal from the serving eNB and neighbor eNBs, as shown in Figure 1. The eNB is set close to the track with a distance of $a$. The foot point is defined as point $\mathrm{O}$, and the distance between point $\mathrm{O}$ and the first radio transceiver is $b$. 


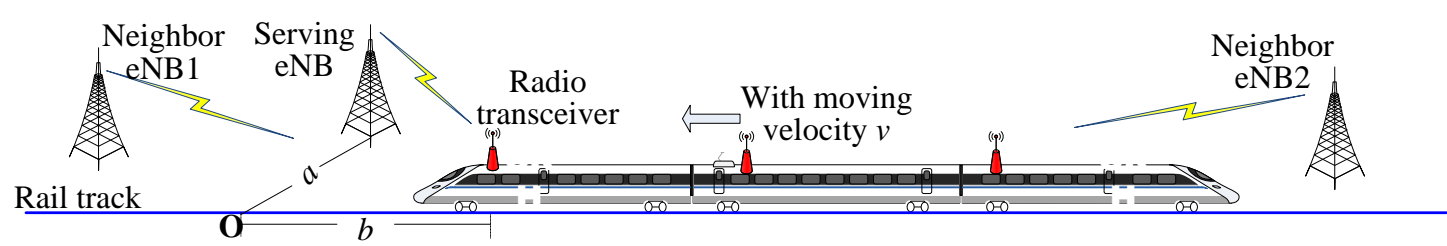

Figure 1. An Example of Moving Train

When the train is moving toward $\mathrm{O}$ on the track with velocity $v$, the Doppler shift for the radio transceiver is incurred by fast moving train. The information about Doppler shift is the phase shift which is introduced in each received symbol. Doppler shift caused by fast moving train, will distort the received signal and incur the train position error. For enough information about Doppler frequency offset estimation, pilots are inserted into separated places of a subframe, as shown in Figure 2.

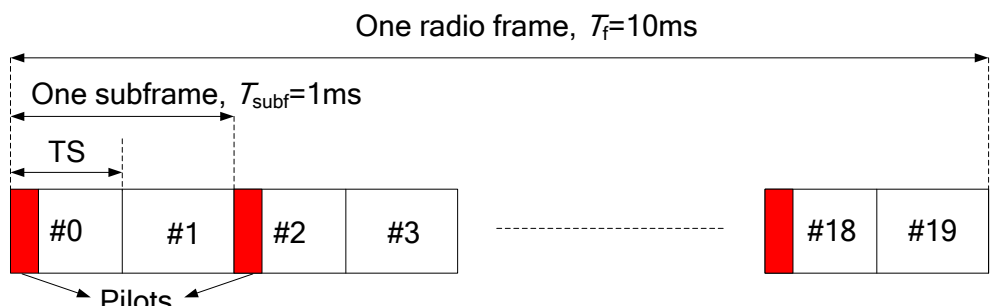

Figure 2. LTE Frame Configuration and Pilots

One element shared by the LTE downlink/uplink is the generic frame structure. The LTE specifications define both FDD and TDD modes of operation. This generic frame structure is used with FDD. Alternative frame structures are defined for use with TDD. In the generic frame structure, LTE radio frames are $10 \mathrm{~ms}$ in duration, which are divided into 10 subframes, and each subframe is $1 \mathrm{~ms}$ long. Each subframe is further divided into two slots, each of $0.5 \mathrm{~ms}$ duration. Slots consist of either 6 or 7 OFDM symbols, depending on whether the normal or extended cyclic prefix is employed.

Assuming $K$ pilots putted into $N$ receive symbols [13], so there will be $(N-K) / K$ symbols after each pilot. The ratio $(N-K) / K$ is represent as the transmission efficiency, $\eta$. There is certainly a trade-off between $\eta$ and Doppler shift estimation accuracy, as well as computation complexity. A frame or subframe need to be designed properly to make sure the Doppler shift won't change in one subframe. The high speed train is commonly moving at a speed of nearly $100 \mathrm{~m} / \mathrm{s}$, so if we have $f_{c}=2 \mathrm{GHz}$, then the coherent time will be $c /(f v) \approx 1.5 \mathrm{~ms}$. One LTE subframe is $1 \mathrm{~ms}$ duration to minimize the effect of Doppler shift.

\subsection{Channel Model}

The radio transceivers on the train received the PRS transmitted by eNB to evaluated train position. The PRS are sent in a configurable number of consecutive subframes, which could be just one subframe or as many as 5 subframes. Since the LTE downlink signal is based on the OFDM modulation within a subframe containing the PRS. The PRS are transmitted on more subcarriers and more OFDM symbols when compared to the regular cell-specific reference signals being sent on an antenna. PRS can be expressed by

$$
x_{l}^{i}(n)=\frac{1}{N} \sum_{k \in S_{\mathrm{PRS}}^{i}} X_{l}^{i}[k] \cdot e^{\mathrm{j} 2 \pi n k / N} \quad\left(-N_{\mathrm{CP}} \leq n<N\right)
$$

where $l$ is the OFDM symbol number, $i$ is the eNB number, $S_{\mathrm{PRS}}^{i}$ is the carrier set of $i$-th eNB, $N$ is the FFT block size, and $N_{\mathrm{CP}}$ is the value of cyclic prefix. 
All radio transceivers on the train can receive signal. The received signal suffers from Ricean fading $h_{p}$, a power loss $1 / l_{p}^{\alpha}$, a Doppler shift $f_{p}$ and an added white Gaussian noise $w_{p}(n)$ with a variance of $2 \sigma^{2}$. We sample the base band received signal with sampling period $T_{s}$, and then, the received signal $y_{p}(n)$ can be written as

$$
y_{p}(n)=x\left(n-\tau_{p}\right) \cdot \frac{h_{p}}{l_{p}^{\alpha}} \cdot e^{j 2 \pi f_{p} n T_{s}}+w_{p}(n)
$$

where $p$ ranges from 0 to $P-1$, representing total $P$ radio transceivers on the train, and $\tau_{p}$ is the time delay of $p$-th radio transceiver. Let the length of the train carriage be $l$, which is typically long enough for added noise for each transceiver to be irrelevant. Then, we get

$$
l_{p}=b+p l
$$

Actually, an accurate estimation of the distance $b$ can be given out with some cutting-edge equipment, such as laser ranging equipment.

The Ricean fading effect $h_{p}$ can be expressed by

$$
h_{p}=\sqrt{\frac{\kappa}{\kappa+1}} e^{j \varphi_{p}}+\sqrt{\frac{1}{\kappa+1}} z_{p}=r_{p} e^{j \phi_{p}}
$$

where $z_{p} \sim \mathrm{CN}(0,1), \kappa$ is the so-called Ricean factor, $r_{p}$ obeys Ricean distribution with the center 1 , and the random phase shift $\phi_{p}$ is evenly distributed in $[0,2 \pi)$. The PDF of all $r_{p}$ is given as

$$
f(r)=2(\kappa+1) \operatorname{rexp}\left(-\frac{r^{2}+\kappa /(\kappa+1)}{1 /(\kappa+1)}\right) I_{0}(2 \sqrt{\kappa(\kappa+1)} r)
$$

In $(5), I_{0}(\cdot)$ is the zero order Bessel function.

\section{Moving Train Position Scheme}

\subsection{Channel Model Doppler Shift Offset Estimation}

For each subframe, we assume that $K$ pilots be used as the Doppler shift estimation. We consider radio transceiver $p$ and represent the whole received signals $y_{p}(k)$ for pilots $x(k)$ as

$$
y_{p}(k)=x\left(k-\tau_{p}\right) \cdot \frac{h_{p}}{l_{p}^{\alpha}} \cdot e^{j 2 \pi f_{p} k \frac{N}{K} T_{s}}+w_{p}(k)
$$

here $k$ ranges from 0 to $K-1$. Furthermore, we define

$$
A_{p}=\frac{r_{p}}{l_{p}^{\alpha}}, B_{P}(k)=2 \pi f_{p} k \cdot \frac{N}{K} T_{s}
$$

We take $x(k)=1$ for each pilot, then (6) can be simplified as

$$
y_{p}(k)=A_{p} e^{j B_{p}(k)+\phi_{p}}+w_{p}(k)
$$

The Doppler shift for each radio transceiver is defined as

$$
\begin{gathered}
f_{p}(t)=\frac{f \cdot v}{c} \cos \theta_{p}(t) \\
\cos \theta_{p}(t)=\frac{l_{p}-v t}{\sqrt{\left(l_{p}-v t\right)^{2}+a^{2}}}, p=0 \sim P-1
\end{gathered}
$$


To estimate each $f_{p}$, the only thing that would stay unknown for us is velocity $v$. However, the velocity estimation remains a very difficult problem for mobile communication, as is shown in [14-16]. In fact, let $y_{p}^{R}(k)$ and $y_{p}^{I}(k)$ are real and imaginary part of $y_{p}(k)$, and the estimation for velocity $v$ by MLE algorithm is given as [13]

$$
\begin{gathered}
\hat{v}=\arg \max \frac{-1}{\sigma^{2}} \sum_{p=0}^{P-1} \sum_{k=0}^{K-1}\left[y_{p}^{R}(k)-A_{p} \cos \left(B_{p}(k)+\phi_{p}\right)\right]^{2} \\
+\left[y_{p}^{I}(k)-A_{p} \sin \left(B_{p}(k)+\phi_{p}\right)\right]^{2} \\
=\arg \max \sum_{p=0}^{P-1} \sum_{k=0}^{K-1} y_{p}^{R}(k) A_{p} \cos \left(B_{p}(k)+\phi_{p}\right) \\
+y_{p}^{I}(k) A_{p} \sin \left(B_{p}(k)+\phi_{p}\right) \\
=\arg \max \operatorname{Re}\left[\sum_{p=0}^{P-1} A_{p} \sum_{k=0}^{K-1} y_{p}(k) e^{-j\left(B_{p}(k)+\phi_{p}\right)}\right]=\arg \max \operatorname{abs}\left[\sum_{p=0}^{P-1} A_{p} \sum_{k=0}^{K-1} y_{p}(k) e^{-j B_{p}(k)}\right]
\end{gathered}
$$

Once the velocity $v$ is determined, we can calculate each $f_{p}$ directly by (9) and (10).

\subsection{Moving Train Position}

The existence of the PRS enables the UE to make OTDOA measurements. According to the principle of correlation detection, the time shift $\tau_{p}$ between the PRS reference sequence and $x\left(n-\tau_{p}\right)$ is generally accepted as the maximum of the cross correlation function. By comparing the values of $\tau_{p}$ from multi-paths, we can get the PRS arrival time of the shortest path between the reference eNB and moving train, i.e.,

$$
\tau=\min \tau_{p}
$$

Assuming the eNB1 and eNB2 are position references, the PRS time difference of arrival can be given by

$$
\tau_{\mathrm{TDOA}}=\left|\tau_{1}-\tau_{2}\right|
$$

where $\tau_{1}$ and $\tau_{2}$ are the PRS arrival time of eNB1 and eNB2, respectively. For the moving train, the values of $\tau_{1}$ and $\tau_{2}$ are closely related to the specific railway alignment. There are usually three types of railway alignment, i.e., straight line, circle curve, and cubic parabola transition curve. The train running curve, $f(x, y)$ can be determined by inquiring about the line database of track section, according to the information of train interlocking route.

If the train runs in a straight line, let $d_{1}$ and $d_{2}$ are the distances between the eNB 1 and moving train, and eNB2 and moving train, respectively, $\left(x_{1}, y_{1}\right)$ and $\left(x_{2}, y_{2}\right)$ are the position coordinates of eNB1 and eNB2, respectively, then the PRS arrival time from the position reference eNB1 and eNB2 are

$$
\tau_{1}=d_{1} / c=1 / c \times \sqrt{\left(x-x_{1}\right)^{2}+\left(y-y_{1}\right)^{2}}
$$

and

$$
\tau_{2}=d_{2} / c=1 / c \times \sqrt{\left(x-x_{2}\right)^{2}+\left(y-y_{2}\right)^{2}}
$$

where, $(x, y)$ is the position coordinate of moving train to be calculated. 
However, if the train runs in a circle curve or transition curve, the radio remote unit and leaky cable are the commonly coverage manners, then, the wireless signal will not travel in straight lines, illustrated in Figure 3. Let $d$ ' be the wireless transmission distance, and $r$ is the radius of circle curve, then the linear distance between the position reference eNB and moving train is

$$
d=2 r \sin \frac{90 d^{\prime}}{\pi r}
$$

Actually, Eq. (16) can be used as the non line of sight (NLOS) distance correction for the curved track section, which obeys chained and redundant macro-cell coverage planning.

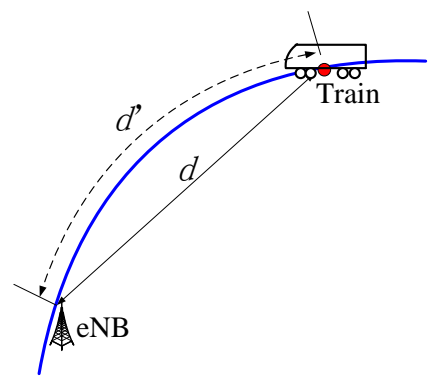

\section{Figure 3. Example of Wireless Transmission in Leaky Cable Coverage Manner}

The PRS time difference of arrival from eNB1 and eNB2, corresponds to a unilateral hyperbolic, $\tau_{\text {TDOA }}$ which is expressed by

$$
\begin{aligned}
& \tau_{\mathrm{TDOA}}=g(x, y)=\left|\tau_{1}-\tau_{2}\right|=1 / c \times\left|d_{1}-d_{2}\right| \\
& =1 / c \times\left|\sqrt{\left(x-x_{1}\right)^{2}+\left(y-y_{1}\right)^{2}}-\sqrt{\left(x-x_{2}\right)^{2}+\left(y-y_{2}\right)^{2}}\right|
\end{aligned}
$$

Then, the intersection of two curves, $g(x, y)$ and train moving trajectory $f(x, y)$, will be the train position illustrated in Figure 4.

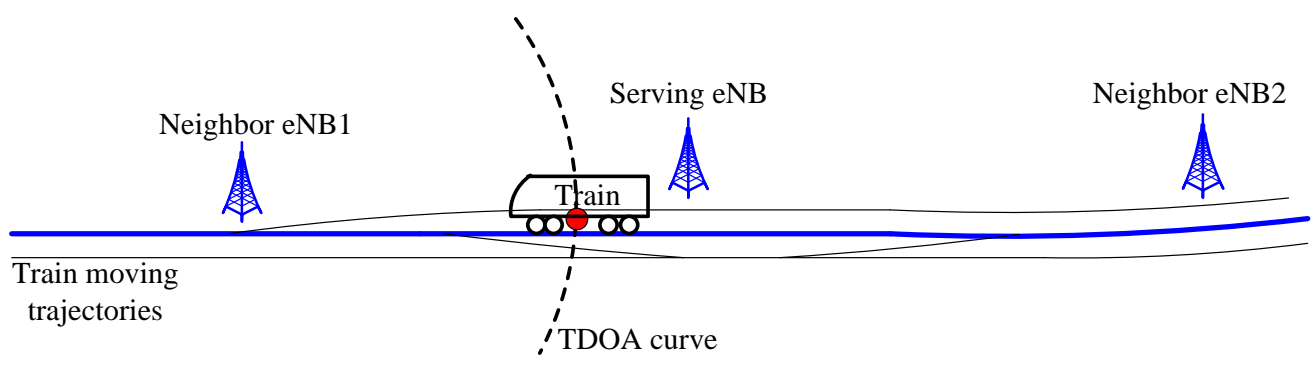

Figure 4. Train Position Calculation

Note that, if $g(x, y)$ and $f(x, y)$ are accurate, and if the other assumptions are ideal, then $(x$, $y$ ) should be the real position coordinate of moving train, i.e, intersection is one. However, there is uncertainty for PRS wireless transmission, and moreover, the train may not travel at a constant velocity, the intersection of two curves may be none or multiple points. The variation of the train velocity is not acute for a short time due to the ride quality of highspeed train. Therefore, the train position can be predicted by the historical data value. That is, if the intersection of two curves is none, the predicted value as real data, and, if the intersection of two curves exists multiple points, the nearest point from the predicted value as real data. Although the turning radius of rail is large for high-speed train, the 
train can be regarded as moving in a straight line for a very short period of time, and then the predicted position coordinates of train, $\left(x_{t+1}, y_{t+1}\right)$ can be obtained as

$$
\left\{\begin{array}{l}
x_{t+1}=x_{t}+s \cos \theta \\
y_{t+1}=f\left(x_{t+1}\right)
\end{array}\right.
$$

where, $\theta$ is the rotation angle, and $s$ is given by

$$
s=\sqrt{\left(x_{t}-x_{t-1}\right)^{2}+\left(y_{t}-y_{t-1}\right)^{2}}
$$

\section{Simulation}

In this section, we demonstrate the positioning performance, with the emphasis on the effect of the estimated Doppler shift on the positioning accuracy. In the simulation, we set $f_{c}=2 \mathrm{GHz}$ and each eNB is deployed every $2 \mathrm{~km}$ or so. Also, it is assumed that there are 4000 symbols for each subframe and 50 symbols among them used as pilots to estimate the Doppler shift. The OFDM transceiver platform is built up according to the LTE-R9 specification, shown in Table.1. Moreover, two types of railway alignments, i.e., straight line and circle curve are considered, illustrated as Figure 5.

Tabel 1. OFDM Parameters

\begin{tabular}{ll}
\hline Parameters & Values \\
\hline Duration of one LTE & $1 \mathrm{~ms}$ \\
subframe & \\
CP length & normal \\
Number of OFDM symbols & 7 \\
FFT size & 2048 \\
Bandwidth & $10 \mathrm{MHz}$ \\
Sampling frequency & $15.36 \mathrm{MHz}$ \\
PRB occupied by PRS & 60 \\
\hline
\end{tabular}

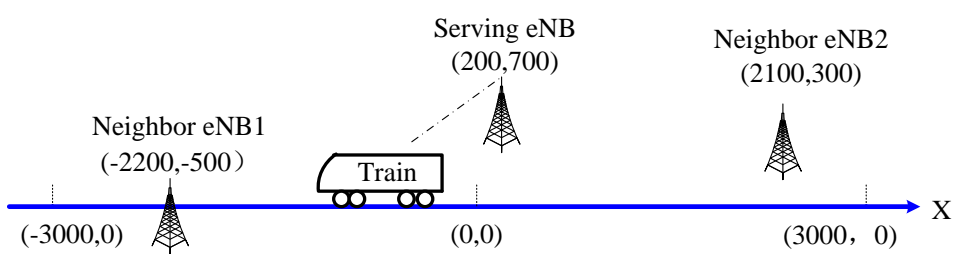

(a) Straight line alignment

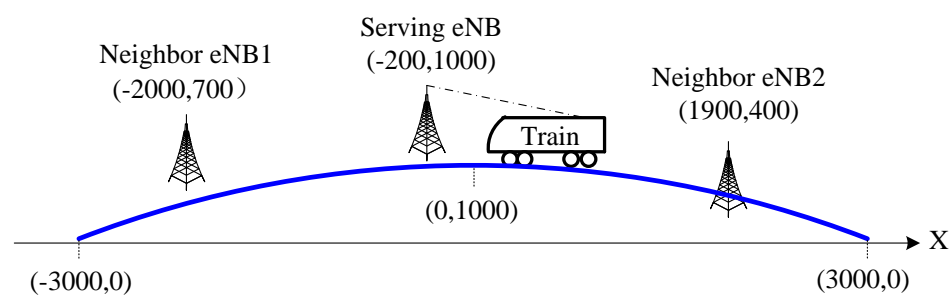

(b) Circle curve alignment

\section{Figure 5. Railway Alignments Setting}

In order to assess the positioning accuracy of the proposed approach, the positioning error, i.e., root mean squared error (RMSE) is introduced which is defined as

$$
R M S E=\sqrt{E\left[(x-\hat{x})^{2}+(y-\hat{y})^{2}\right]}
$$


where $(x, y)$ and $(\hat{x}, \hat{y})$ are the correct and estimated coordinates of the moving train, respectively.

Figure 6 and Figure 7 show the impact of bias of correct and estimated Doppler shift ( $\Delta f=f_{p}-\hat{f}_{p}$ ) on position error (RMSE) for the different train moving trajectories. From the figures, we can see that with the increasing bias of the correct and estimated Doppler shift, the positioning error RMSE increases accordingly. Also, it can be observed that the straight line alignment has the better position accuracy for the moving train, compared to the circle curve alignment.
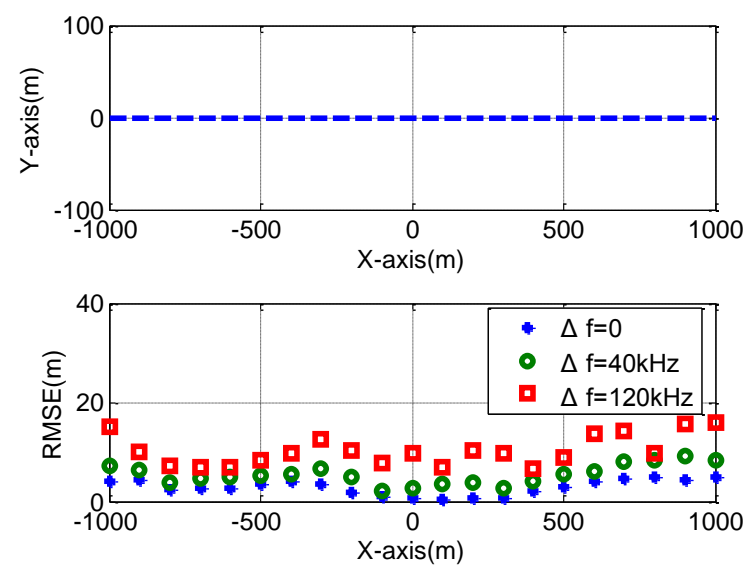

Figure 6. Positioning Error RMSE when the Train Moves in Straight Line (SNR=18dB)
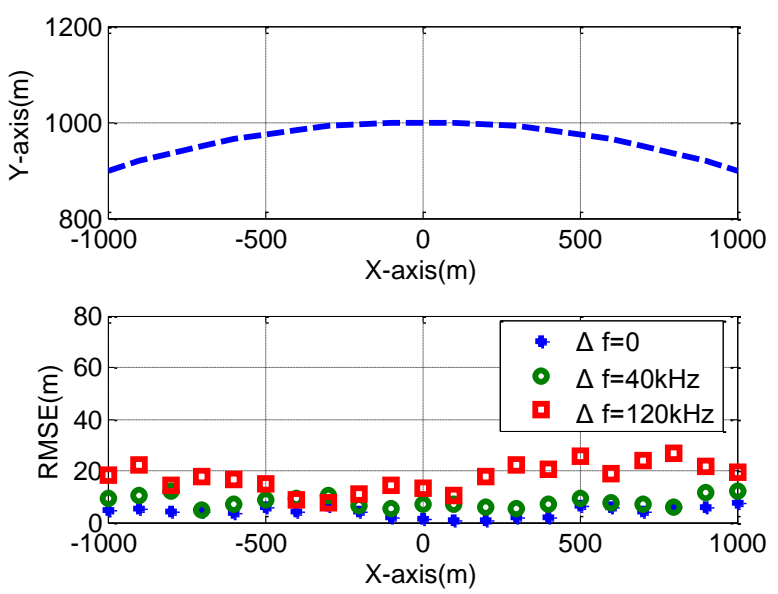

\section{Figure 7. Positioning Error RMSE when the Train Moves in Circle Curve} $(S N R=18 \mathrm{~dB})$

\section{Conclusions}

This paper has proposed a PRS-oriented localization approach for railway vehicles. The PRS are sent in a configurable number of consecutive subframes and the Doppler shift is estimated based on the pilots in each subframe. The subsequent incorporation of the PRS time difference of arrival from positioning references eNB and the train moving trajectory is investigated to predict the position coordinates of train. Simulation results have shown the good performance of the positioning accuracy. Future work will focus on the effects of the variation of the train velocity 
and the turning radius of rail in high-speed railway, on the Doppler shift estimation error and train position accuracy.

\section{Acknowledgements}

This work was partially supported by Nation Science Foundation of China (61261014), and Natural Science Foundation of Gansu Province, China (148RJZA037).

\section{References}

[1] N. Burton, "Auckland metropolitan resignalling project: Comprehensive modernization", CORE 2010: Rail, Rejuvenation and Renaissance, Engineers Australia (2010), p. 707.

[2] R. Mazl and L. Preucil, "Sensor data fusion for inertial navigation of trains in GPS-dark areas", Proceedings of IEEE Intelligent Vehicles Symposium, (2003).

[3] Z. D. Zhong, B Ai, Q. Y. Liu and S. Y. Lin, "GSM-R Application Fundamental Theory, Tsinghua University Press and Beijing Jiaotong University Press", (2009).

[4] UIC, LTE/SAE-The Future Railway Mobile Radio System? Long-Term Visions on Railway Mobile Radio Technologies, V 0.4 Draft. 14.09, (2009).

[5] 3GPP, Evolved Universal Terrestrial Radio Access (E-UTRA); physical channels and modulation, 3GPP TS 36.211, version 9.1.0 Release 9, (2009).

[6] 3GPP, Evolved Universal Terrestrial Radio Access (E-UTRA); LTE Positioning Protocol (LPP), 3GPP TS 36.355 V9.4.0, (2010).

[7] J. Medbo, I. Siomina. A. Kangas and J Furuskog, "Propagation channel impact on LTE positioning accuracy", Proceedings of the $20^{\text {th }}$ IEEE International Symposium on Personal, Indoor and Mobile Radio Communication, (2009).

[8] T. Zhang, D.Xiao, J. Cui and X. Luo, "A novel OTDOA positioning scheme in Heterogeneous LTEAdvanced systems", Proceedings of 3rd IEEE International Conference on Network Infrastructure and Digital Content, (2012).

[9] R. M. Vaghefi and R M.Buehrer, "Improving positioning in LTE through collaboration", Proceedings of the 11th IEEE Workshop on Positioning, Navigation and Communication, (2014).

[10] S. Bohanudin, M. Ismail, and H. Hussain, "Simulation model and location accuracy for observed time difference of arrival (OTDOA) positioning technique in third generation system", Proceedings of the IEEE Student Conference on Research and Development, (2010).

[11] L. H. Yang, G. L. Ren and Z. L. Qiu, "A Novel Doppler Frequency Offset Estimation Method for DVBT System in HST Environment. IEEE Transactions on Broadcasting", vol. 58, no. 1, (2012).

[12] E. P. Simon, L. Ros, H. Hijazi and M. Ghogho, "Joint Carrier Frequency Offset and Channel Estimation for OFDM Systems via the EM Algorithm in the Presence of Very High Mobility", IEEE Transactions on Signal Processing, vol. 60, no. 2, (2012).

[13] Y. Q. Yang, P. Y. Fan and Y. M. Huang, "Doppler frequency offsets estimation and diversity reception scheme of high speed railway with multiple antennas on separated carriages", Proceedings of the IEEE International Conference on Wireless Communications \& Signal Processing, (2012).

[14] A. G. Zajic, "Estimation of Mobile Velocities and Direction of Movement in Mobile-to-Mobile Wireless Fading Channels", IEEE Transactions on Vehicular Technology, vol. 61, no. 1, (2012).

[15] C. Tepedelenlioğlu and G. B. Giannakis, "On Velocity Estimation and Correlation Properties of Narrow-Band Mobile Communication Channels", IEEE Transactions on Vehicular Technology. Vol. 50, no. $4,(2001)$.

[16] Y. R. Zheng and C. S. Xiao, "Mobile Speed Estimation for Broadband Wireless Communications over Rician Fading Channels", IEEE Transactions on Wireless Communications, vol. 8, no. 1, (2009).

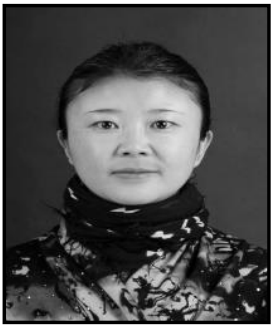

Cuiran Li, she received a BSc. and Master degree from the Department of Communication Engineering, Lanzhou Jiaotong University, in 1996 and 1999, respectively, and a Dr. degree from the School of Electronics and Information Engineering, Beijing Jiaotong University in 2003. She is now working in Lanzhou Jiaotong University as a professor and an IEEE member. She has published a book and over 40 scientific papers in her research area till now. Her 
research interests include cognitive radio techniques, mobile ad hoc network and GSM-R/LTE-R systems.

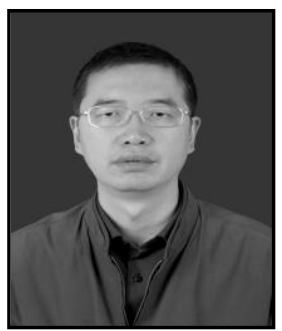

Jianli Xie, he received a BSc. Degree from Sichuan University in 1993, and a Master degree and Dr.degree from the School of Electronics and Information Engineering, Lanzhou Jiaotong University in 1999 and 2014, respectively. From 1999-2007, he was a senior hardware engineer in Beijing Great Dragon company. Now he is working in Lanzhou Jiaotong University as an associate professor. He has published over 20 scientific papers in his research area till now. His research interests include wireless communication network and cognitive radio techniques. 